\title{
A novel route for the inclusion of metal dopants in silicon
}

\author{
Jules A Gardener ${ }^{1,7}$, Irving Liaw ${ }^{2,8}$, Gabriel Aeppli ${ }^{1}$, \\ Ian W Boyd ${ }^{2,9}$, Richard J Chater ${ }^{3}$, Tim S Jones ${ }^{4}$, \\ David S McPhail ${ }^{3}$, Gopinathan Sankar ${ }^{5}$, A Marshall Stoneham ${ }^{1}$, \\ Marcin Sikora $^{6,10}$, Geoff Thornton ${ }^{5}$ and Sandrine Heutz ${ }^{3}$ \\ ${ }^{1}$ Department of Physics and London Centre for Nanotechnology, University College London, \\ London WC1E 6BT, UK \\ ${ }^{2}$ Department of Electronic and Electrical Engineering and London Centre for \\ Nanotechnology, University College London, London WC1E 6BT, UK \\ ${ }^{3}$ Department of Materials and London Centre for Nanotechnology, Imperial College London, \\ London SW7 2AZ, UK \\ ${ }^{4}$ Department of Chemistry, University of Warwick, Coventry CV4 7AL, UK \\ ${ }^{5}$ Department of Chemistry and London Centre for Nanotechnology, University College \\ London, London WC1E 6BT, UK \\ ${ }^{6}$ ESRF, 6 rue Jules Horowitz, BP 220, F-38043 Grenoble Cedex, France \\ E-mail: s.heutz@imperial.ac.uk
}

Received 27 August 2009, in final form 27 October 2009

Published 3 December 2009

Online at stacks.iop.org/Nano/21/025304

\begin{abstract}
We report a new method for introducing metal atoms into silicon wafers, using negligible thermal budget. Molecular thin films are irradiated with ultra-violet light releasing metal species into the semiconductor substrate. Secondary ion mass spectrometry and x-ray absorption spectroscopy show that $\mathrm{Mn}$ is incorporated into $\mathrm{Si}$ as an interstitial dopant. We propose that our method can form the basis of a generic low-cost, low-temperature technology that could lead to the creation of ordered dopant arrays.
\end{abstract}

(Some figures in this article are in colour only in the electronic version)

Chemically versatile, minimal thermal budget approaches to the controlled doping of semiconductor wafers become increasingly important as feature sizes fall. Subjecting pre-patterned dopant regions to high temperatures enhances diffusion and is detrimental to interface definition. Ion implantation or metallic thin film precursors can be used to dope semiconductors, but these procedures rely on annealing to produce high quality wafers $[1,2]$. Alternatively, growth methods such as molecular beam epitaxy (MBE) or metal organic chemical vapour deposition (MOCVD) can be utilized, although these are expensive and also require a high-

7 Present address: Department of Physics, Harvard University, Cambridge, MA 02138, USA.

8 Present address: School of Chemistry, University of Melbourne,

Victoria 3010, Australia.

9 Present address: Melbourne Centre for Nanofabrication, Monash University, VIC 3800, Australia.

10 Present address: Department of Solid State Physics, AGH University of Science and Technology, 30-059 Krakow, Poland. temperature step [3-5]. Lateral control of dopant positioning can be achieved using scanning probe methods [6, 7] or, recently, annealing of chemisorbed organic films [8]. However, these approaches are limited by a low prospect of upscaling and restrictive dopant-substrate combinations respectively. Here, we demonstrate a new approach, using clean, cheap vacuum ultra-violet (UV) sources [9] to degrade metalorganic thin films on $\mathrm{Si}$ at temperatures close to room temperature. This releases the metal atoms, which are subsequently incorporated into the substrate as dopants.

The molecules chosen are phthalocyanines (MPc, figure 1 inset), which can form ordered films onto virtually any substrate [10]. Once deposited, these molecules remain intact, and so the ligands act to spatially separate the metal atoms. Important for the present application, MPcs can contain a vast range of species at their centre (most transition metals, rare earths, oxides, etc), and we envisage that the doping method demonstrated here for $\mathrm{Mn}$ in Si could be easily generalized to any dopant/substrate combination. Here, we focus on 


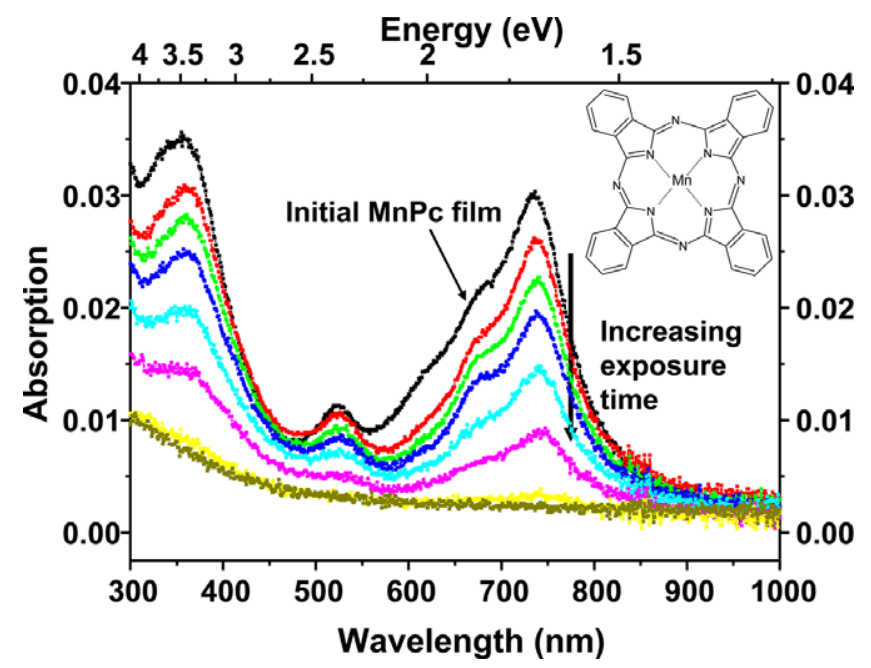

Figure 1. UV-vis spectra of a $5 \mathrm{~nm}$ thick MnPc film on glass (as indicated) and after $172 \mathrm{~nm}$ UV exposure for $30 \mathrm{~s}, 1,2,5,10,20$ and $40 \mathrm{~min}$. Spectra of clean glass substrates subjected to the same UV dosage have subtracted from the raw data to correct for UV-induced changes in each case. The intensity of the MnPc absorption peaks are seen to decrease with increasing UV dosage, demonstrating degradation of the molecular film. The structure of MnPc is shown in the inset.

manganese phthalocyanine $(\mathrm{MnPc})$ to make $\mathrm{Si}: \mathrm{Mn}$, a system with potential applications in spintronics [11, 12].

A B-doped $\operatorname{Si}(100)$ substrate $(0.010 \Omega \mathrm{cm})$ was hydrogen passivated by immersing in $\mathrm{HF}$ (VWR, 50\%) to remove the native oxide layer and immediately loaded into a Kurt J Lesker vacuum deposition chamber (base pressure $8 \times$ $10^{-8}$ mbar). Manganese (II) phthalocyanine (Aldrich), which had been further purified by two cycles of gradient sublimation, was sublimed at $390-400{ }^{\circ} \mathrm{C}$, producing $\alpha$-phase crystalline films wherein the MnPc lie almost perpendicular to the substrate [13]. The final film thickness of $5 \mathrm{~nm}$ was monitored by an in situ quartz crystal microbalance. The films were irradiated with $172 \mathrm{~nm}$ photons from a purpose-built array of four Xe excimer lamps, in a separate chamber backfilled to $5.0 \mathrm{mbar}$ with nitrogen [9]. During irradiation (40 min, unless otherwise stated), an in situ thermocouple showed the substrate remained at $25-60^{\circ} \mathrm{C}$. UV-vis analyses were performed on irradiated MnPc films of glass substrates (to allow optical transmission) using a Perkin-Elmer Lambda 950 UV-vis spectrometer. Secondary ion mass spectroscopy (SIMS) was performed on Si samples with an Atomika 6500 microprobe $\left(1.25 \mathrm{keV}, 30 \mathrm{nA} \mathrm{Ar}{ }^{+}\right.$primary ion beam incident at $45^{\circ}$ to the surface), whilst electron beam compensation was not required. X-ray absorption spectroscopy (XAS) data at the Mn K-edge were collected at the European Synchrotron Radiation Facility (Station ID26) in fluorescence mode using a 13 element Ge detector and $0.9 \mathrm{eV}$ excitation energy resolution. After UV irradiation, a Piranha clean removed any Mn-rich surface residue prior to SIMS and XANES analyses, resulting in a uniform surface $\mathrm{SiO}_{2}$ layer.

MPcs are efficient light absorbers due to $\pi-\pi *$ transitions corresponding to excitation from the molecular ground state to the first and second excited states in the visible and
near-UV regions respectively [14]. UV-vis measurements allow us to monitor the rate at which the MnPc film is degraded during exposure to the UV lamp array, as shown in figure 1. The spectrum of the initial $\mathrm{MnPc}$ film agrees with previous work [15], confirming its integrity before UV exposure. Upon increasing irradiation, the intensity of the characteristic MnPc absorption spectrum decreases in a controlled manner until after forty minutes it is no longer detectable, thus demonstrating that the molecules have been modified and/or removed. The disappearance of the absorption profile corresponds to photo-decomposition [16], as processing temperatures remain below $60^{\circ} \mathrm{C}$ and so are insufficient to sublime the molecules or induce structural changes [17]. From energetic considerations, the first dissociation step is the rupturing of the lowest energy $\mathrm{C}-\mathrm{N}$ bonds, resulting in the formation of pyrrole- and benzene-rich fragments, which themselves are further decomposed by the $172 \mathrm{~nm}$ photons [18]. This destabilizes the metal-ligand bond leading to the release of the metal atoms, whilst other UV-assisted mechanisms aid transfer to the substrate, as discussed below.

Further analysis of the chemical composition of the surface and bulk Si after forty minutes of UV exposure has been performed using SIMS. Initial studies showed a high Mn concentration accompanied with oxygen enrichment before the substrate was reached, followed by a decreasing manganese profile within the silicon. This is consistent with surface manganese oxide, in agreement with other reports [16] and the broad UV-vis peak below $500 \mathrm{~nm}$ (figure 1) [19]. Therefore, to avoid introduction of $\mathrm{Mn}$ due to beam-induced mixing [20], and to focus on the $\mathrm{Mn}$ that has been incorporated into $\mathrm{Si}$ exclusively by the UV process, the UV-irradiated samples were Pirhana cleaned to remove any Mn-rich surface contaminants. Figure 2(a) shows a sample that has been UV-treated and subjected to a Piranha clean. Even after cleaning, we observe a stable Mn presence which extends for depths of up to $95 \mathrm{~nm}$. Numerous analyses were performed on the bare wafer (for example, as shown in figure 2(b)) and on Pirhana cleaned asdeposited MnPc films, none of which showed any traces of Mn. This confirms that the UV (rather than chemical) process leads to $\mathrm{Mn}$ incorporation. We estimate a Mn concentration of $7 \times$ $10^{20}$ atoms $\mathrm{cm}^{-3}$ from the SIMS profile in figure 2(a) using the relative sensitivity factors [21]. Spatial composition mapping highlights lateral inhomogeneities and figure 2(a) represents the maximum Mn concentration observed. The concentration of $\mathrm{Mn}$ atoms in a MnPc film can be approximated by assuming that the unit cell of MnPc is similar to that of $\alpha$-phase $\mathrm{CuPc}$, with dimensions $a=25.9 \AA, b=3.8 \AA, c=23.9 \AA$ and $\beta=90.4^{\circ}$ (with four metal atoms per unit cell) [22]. This concentration $\left(C_{\mathrm{MnPc}}\right)$ is calculated in equation (1), where $V_{\mathrm{uc}}$ is the volume of the unit cell $\left(\right.$ in $\mathrm{cm}^{3}$ ).

$$
C_{\mathrm{MnPc}}=\frac{4}{V_{\mathrm{uc}}}=\frac{4}{a b c \sin \beta}=1.7 \times 10^{21} \text { atoms cm }^{-3} \text {. }
$$

The number of $\mathrm{Mn}$ atoms in a $1 \mathrm{~cm} \times 1 \mathrm{~cm}$ surface region of a MnPc film of thickness $d_{\text {film }}$ (in cm, in this case $5 \mathrm{~nm}$ thick) is given by equation (2).

$$
N_{\mathrm{Mn}}=C_{\mathrm{MnPc}} d_{\text {film }}=8.5 \times 10^{14} \text { atoms } \mathrm{cm}^{-2} .
$$



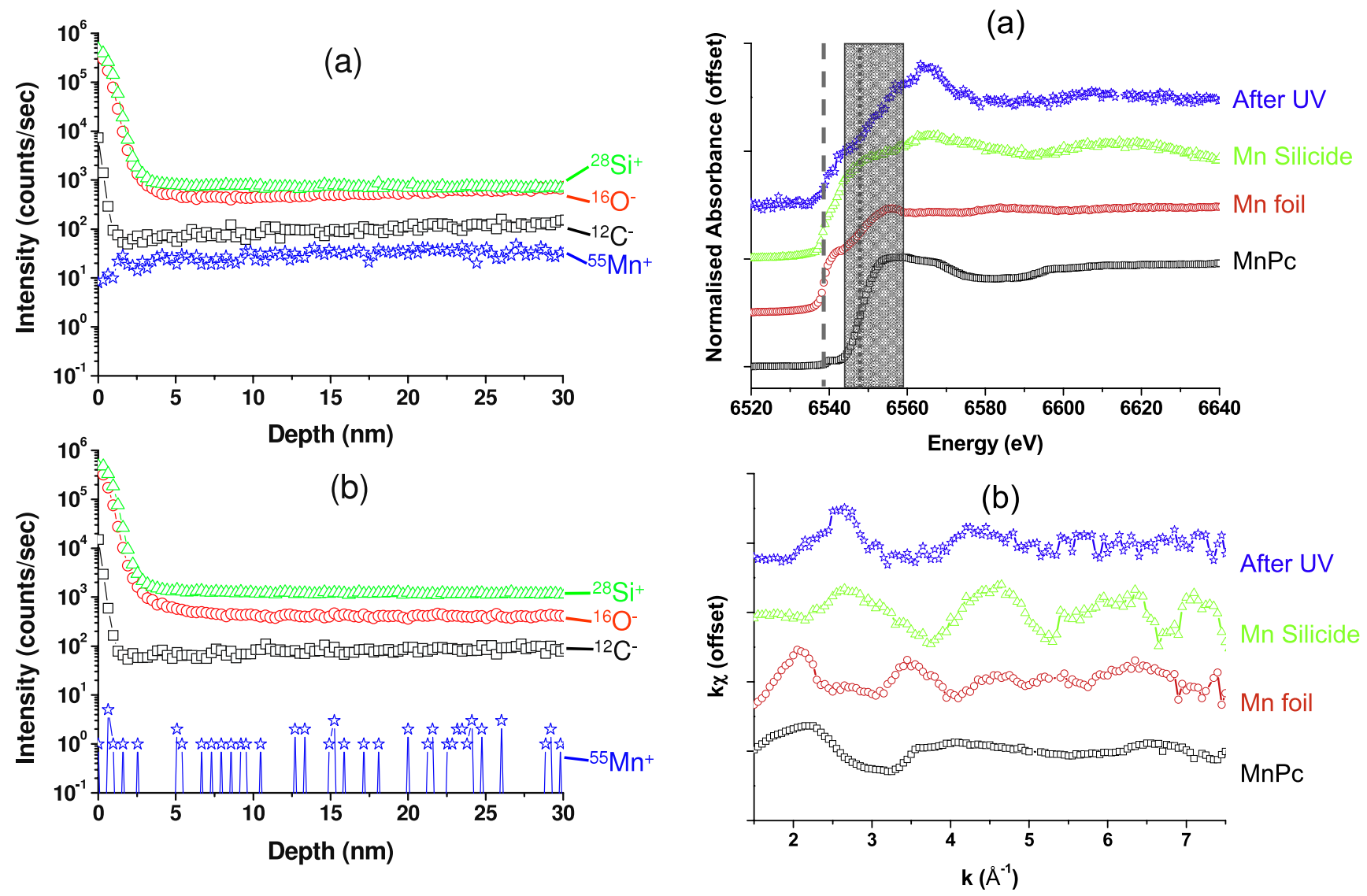

Figure 2. SIMS depth profiles of (a) a $5 \mathrm{~nm} \mathrm{MnPc} \mathrm{film} \mathrm{on} \mathrm{Si} \mathrm{that} \mathrm{had}$ been UV irradiated and Pirhana cleaned, then stored in ultra-high vacuum at room temperature for $25 \mathrm{~h}$ prior to measurement and (b) the initial silicon wafer. A significant quantity of $\mathrm{Mn}$ is observed in the UV exposed sample, which is not present in the original Si wafer.

Deriving the implanted Mn concentration from over 30 analyses over a range of sample preparation runs and comparing to the initial Mn concentration calculated above yields an efficiency for doping of $65 \pm 15 \%$.

The type of bonding of the Mn dopant in the Si lattice can be inferred from the value of the Mn diffusion coefficient, $D$. We estimate the diffusion coefficient using successive SIMS measurements (with time delays of one day) and the solution of Fick's equation, which gives the concentration $C$ of a dopant as a function of depth, $x$, and time, $t$, after diffusion from a surface concentration $C(0)$ as given in equation (3).

$$
C(x, t)=C(0) \operatorname{erfc}\left(\frac{x}{2 \sqrt{D t}}\right) .
$$

We find $D \sim(0.8 \pm 0.1) \times 10^{-15} \mathrm{~cm}^{2} \mathrm{~s}^{-1}$ at room temperature, which is close to the reported value for interstitial $\mathrm{Mn}$ in $\mathrm{Si}$ as determined by deep level transient spectroscopy and the Hall effect $\left(2.8 \times 10^{-15} \mathrm{~cm}^{2} \mathrm{~s}^{-1}\right)$ [23]. We note that the preferential formation of interstitial $\mathrm{Mn}$ is consistent with reports elsewhere [24, 25]. The relatively constant Mn concentration observed in figure 2(a) is contrary to the decrease of $\sim 10 \%$ expected over a depth of $30 \mathrm{~nm}$ using equation (3). This is likely to be due to experimental limitation in the stability of the SIMS measurements and to the beam-induced mixing of surface $\mathrm{Mn}$ into the wafer.

Figure 3. (a) Normalized XANES profiles and (b) EXAFS oscillations for MnPc, Mn, Mn silicide and a MnPc film after UV exposure. Dashed and dotted lines show the edge position for $\mathrm{Mn}^{0}$ and $\mathrm{MnPc}$ respectively, while the shaded box highlights the region of Mn oxides. The spectra for the implanted sample are noisier due to the smaller concentration of $\mathrm{Mn}$ present. The closest agreement is observed between the UV-treated sample and the Mn silicide.

More detailed information about the local chemical environment of the dilute implanted $\mathrm{Mn}$ can be obtained using XAS techniques. The total Mn K-fluorescence yield from a UV-treated MnPc film after cleaning and storing at $193 \mathrm{~K}$, compared with bulk Mn, gives an Mn concentration of $\sim 5 \times 10^{20}$ atoms $\mathrm{cm}^{-3}$, assuming similar $\mathrm{x}$-ray penetration and escape depths, a value which is consistent with our SIMS data. The UV-treated MnPc film, fresh MnPc film, Mn foil and B20 cubic Mn silicide are compared in the X-ray absorption near edge spectroscopy (XANES) profiles shown in figure 3(a). These post-normalization absorption features show notable differences between the UV-treated and precursor films. A significant lowering of the x-ray absorption edge by $\sim 8.5 \mathrm{eV}$ with respect to the precursor film (represented by the dashed and dotted lines respectively) demonstrates that $\mathrm{Mn}$ is no longer bound to the organic ligand, consistent with our UV-vis data (figure 1). The formation of oxides, which typically show an absorption edge shift of 5-15 eV higher than bulk Mn [26] (region highlighted in grey) are also ruled out. Instead, the edge of the UV-treated sample corresponds to $\mathrm{Mn}^{0}$, as it occurs at the same energy as metallic Mn. However, the higher energy $\mathrm{x}$-ray absorption features differ markedly from the Mn foil, so 
the formation of Mn clusters is unlikely. Instead, the lineshape and edge position of the UV-treated sample strongly resemble the silicide reference, suggesting Mn atoms surrounded by Si. This is even more evident in the extended x-ray absorption fine structure (EXAFS) spectrum (figure 3(b)), where the oscillations seen for the silicide and our doped sample occur at similar positions, contrary to what is observed for the metal or MnPc film. Whilst our experimental results have established that the Mn atoms are in a neutral oxidation state surrounded by silicon, it is unlikely that they form pure, stable bulk silicides. This is seen in the subtle discrepancies in the XANES and EXAFS spectra, suggesting differences in the Mn-Si distance and/or coordination number, whilst SIMS imaging shows a low $\mathrm{Mn}: \mathrm{Si}$ ratio $(\sim 1: 70)$ combined with an absence of Mn-rich clusters. Instead, the XANES spectrum agrees very well with that of dilute (isolated) Mn in Si systems, which in turn differs significantly from substitutional Mn:Si [27]. The XAS analysis therefore confirm our interpretation of interstitial doping.

We deduce that once the $\mathrm{Mn}$ atoms are released from the organic macrocycle they reach the $\mathrm{Si}$ substrate and are introduced into its bulk via various photo-assisted mechanisms. Under equilibrium conditions, incorporation of interstitial Mn into Si typically requires $\sim 2.5-3.2 \mathrm{eV}$ [24], which is amply provided by the $172 \mathrm{~nm}(7.2 \mathrm{eV})$ lamps. We note that a thin $\mathrm{SiO}_{2}$ layer re-growth would not block Mn diffusion [28]. Presumably, the macroscopic Mn inhomogeneity arises from surface or bulk inhomogeneities in the original wafer, associated with recombination-enhanced diffusion due to UVgenerated electron-hole pairs during illumination [29]. If so, the effects might be diminished by decreasing the UV exposure time and reducing the precursor film thickness.

A major advantage of our technique is its potential for lateral control of dopant positions at the nano-scale. Different MPc packing arrangements on substrates would lead to linear, nearly square or random molecular templates [30, 31]. Finetuning of dimensions could be achieved by ligand choice, whilst co-depositing different species could extend the scheme to multiply-doped systems. Accurate control over the time of UV treatment would be essential for the creation of arrays: sufficient to incorporate the $\mathrm{Mn}$ atoms but short enough so as not to promote lateral diffusion when illuminated. We note that temperature can also be used to control dopant diffusion. Interstitial $\mathrm{Mn}$ diffuses relatively fast at room temperature, quickly disturbing any ordering: Mn would diffuse by a distance of $d \sim(D t)^{1 / 2}=1 \mathrm{~nm}$ after a time $t$ of $12 \mathrm{~s}$ (using our derived value of $D$ ). However, the low thermal budget means such difficulties could be overcome by sample cooling during and after preparation; the use of dry ice (193 K) would reduce $\mathrm{Mn}$ diffusion to $\sim 1 \mathrm{~nm}$ in a year, whilst even fast diffusing species such as $\mathrm{Cu}$ would take $\sim 200$ years to migrate $1 \mathrm{~nm}$ at $77 \mathrm{~K}$ [32].

In conclusion, we have described a versatile, lowtemperature method to introduce metal dopants into Si. We have shown that a UV source can be used to photodegrade metal phthalocyanine thin films, and through SIMS and XANES measurements we have demonstrated UVinduced introduction of interstitial $\mathrm{Mn}$ into $\mathrm{Si}$. We envisage that this process could be readily extended to other metal/organic/semiconductor combinations. Our procedure is clean, cheap and commercially viable, and so has a large potential for use in the development of nano-electronic devices.

\section{Acknowledgments}

Financial support from the Research Council UK and the Engineering and Physical Sciences Research Council Basic Technology grant (GR/S23506) is gratefully acknowledged. We thank the Royal Society for a Dorothy Hodgkin research fellowship and a Wolfson Research Merit Award. We acknowledge the ESRF for provision of their facilities and P Glätzel and T-C Weng for assistance. We thank Professors $\mathrm{D}$ van der Marel and $\mathrm{C}$ Renner for providing the $\mathrm{MnSi}$ reference sample.

\section{References}

[1] Grimmeiss H G, Janzén E and Skarstam B 1980 J. Appl. Phys. 513740

[2] Reed M L, El-Masry N A, Stadelmaier H H, Ritums M K, Reed M J, Parker C A, Roberts J C and Bedair S M 2001 Appl. Phys. Lett. 793473

[3] Ohno H, Shen A, Matsukura F, Oiwa A, Endo A, Katsumoto S and Iye Y 1996 Appl. Phys. Lett. 69363

[4] Jamieson D N et al 2005 Appl. Phys. Lett. 86202101

[5] Yang X L, Chen Z T, Wang C D, Huang S, Fang H, Zhang G Y, Chen D L and Yan W S 2008 J. Phys. D: Appl. Phys. 41125002

[6] Schofield S R, Curson N J, Simmons M Y, Rueß F J, Hallam T, Obereck L and Clark R G 2003 Phys. Rev. Lett. 91136104

[7] Kitchen D, Richardella A, Tang J-M, Flatté M E and Yazdani A 2006 Nature 442436

[8] Ho J C, Yerushalmi R, Jacobson Z A, Fan Z, Alley R L and Javey A 2008 Nat. Mater. 762

[9] Boyd I W and Zhang J Y 1997 Nucl. Instrum. Methods Phys. Res. B 121349

[10] McKeown N B 1998 Phthalocyanine Materials: Synthesis, Structure and Function (Cambridge: Cambridge University Press)

[11] Bolduc M, Awo-Affouda C, Stollenwerk A, Huang M B, Ramos F G, Agnello G and LaBella V P 2005 Phys. Rev. B 71033302

[12] Wu H, Kratzer P and Scheffler M 2007 Phys. Rev. Lett. 98117202

[13] Heutz S et al 2007 Adv. Mater. 193618

[14] Sharp J H and Abkowitz M 1973 J. Phys. Chem. 77477

[15] Rajesh K R and Menon C S 2001 Mater. Lett. 51266

[16] Otha N and Gomi M 2000 Jpn. J. Appl. Phys. 394195

[17] Heutz S, Bayliss S M, Middleton R L, Rumbles G and Jones T S 2000 J. Phys. Chem. B 1047124

[18] Li Q-R, Gu C-Z, Di Y, Yin H and Zhang J-Y 2006 J. Hazard. Mater. 13368

[19] Dakhel A A 2006 Thin Solid Films 496353

[20] Yang M H, Mount G and Mowat I 2006 J. Vac. Sci. Technol. B 24428

[21] Novak S W and Wilson R G 1991 J. Appl. Phys. 69463

[22] Ashida M, Uyeda N and Suito E 1966 Bull. Chem. Soc. Japan 392616

[23] Nakashima H and Hashimoto K 1991 J. Appl. Phys. 691440

[24] Dalpian G M, da Silva A J R and Fazzio A 2003 Phys. Rev. B 68113310

[25] Chen H, Zhu W, Kaxiras E and Zhang Z 2009 Phys. Rev. B 79235202

[26] Lima F H B, Calegaro M L and Ticianelli E A 2006 J. Electroanal. Chem. $\mathbf{5 9 0} 152$ 
[27] Wolska A, Lawniczak-Jablonska K, Klepka M, Walczak M S and Misiuk A 2007 Phys. Rev. B 75113201

[28] Zhang L and Ivey D G 1991 J. Mater. Res. 61518

[29] Itoh N and Stoneham A M 2001 Materials Modification by Electronic Excitation (Cambridge: Cambridge University Press)
[30] Gardener J, Owen J H G, Miki K and Heutz S 2008 Surf. Sci. $\mathbf{6 0 2} 843$

[31] Upward M D, Beton P H and Moriarty P 1999 Surf. Sci. 44121

[32] Zamouche A, Heiser T and Mesli A 1995 Appl. Phys. Lett. 66631 